\title{
Geodesic Neighborhoods for piecewise affine interpolation of sparse data
}

V. Caselles and G. Facciolo

Universitat Pompeu Fabra

Montevideo - April 6, 2010 


\section{Objective}

Interpolate range measurements (LIDAR or sparse disparity data) incorporating information provided by a photo $u$ of the scene.

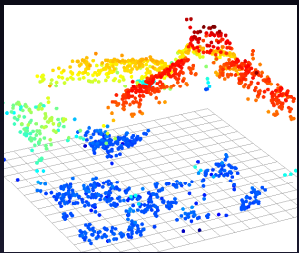

Height samples (5\%)

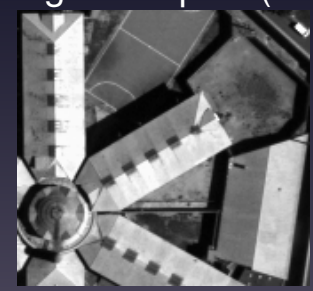

Reference image $u$

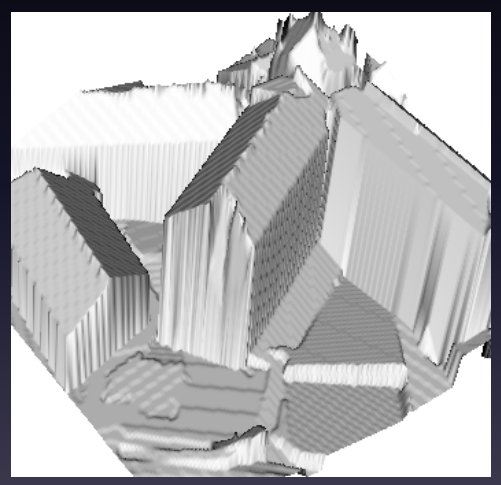

Piecewise affine interpolation 


\section{Previous Work}

\section{Smoothness Prior}

- Polynomial interpolation [Shepard '68]

- Piecewise affine [Preciozzi '05]

- Minimal surface [Facciolo et al. '06]

- AMLE [Almansa et al. '02]

\section{Parametric models}

- Semantic models [Karantzalos \& Paragios '09] [Van Gool et al. '02] 


\section{Geodesic Voronoi Cells}

- Reference Image $u(x): \Omega \rightarrow \mathbb{R}^{+}$, with $\Omega \subset \mathbb{R}^{2}$

- Curve $C(p):[0,1] \rightarrow \Omega$, and $C_{s, t}$ curve connecting $s$ and $t$

- Geodesic distance between $s$ and $t$

$$
d(s, t)=\min _{C_{s, t}} \int_{0}^{1}\left|\nabla u \cdot \dot{C}_{s, t}(p)\right|+\varepsilon\left|\dot{C}_{s, t}(p)\right| d p
$$

discourages paths

- Positions of the samples $\wedge \subset \Omega$

- Depth values of samples $G(\lambda): \Lambda \rightarrow \mathbb{R}, \lambda \in \Lambda$

- Geodesic Voronoi diagram of the sites in $\Lambda$ is defined with the geodesic distance

- Computed with fast marching [Yatziv, Bai, Sapiro '06-'07] 


\section{Geodesic Voronoi Cells}

Geodesic Voronoi diagram of the sites in $\Lambda$, successfully accounts for discontinuities in the image.

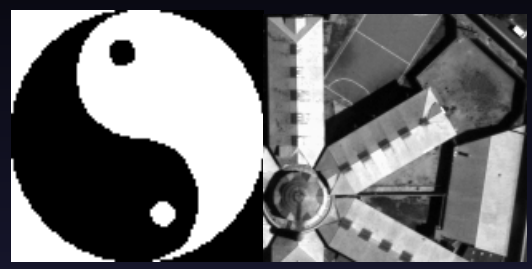

Reference images

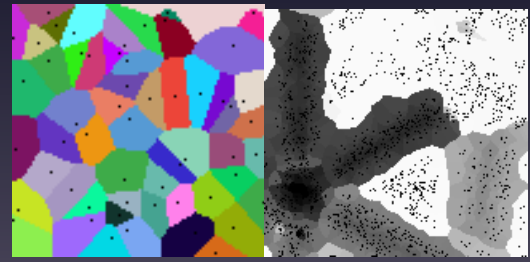

Samples \& Voronoi cells 


\section{Geodesic Voronoi Cells}

Geodesic Voronoi diagram of the sites in $\Lambda$, successfully accounts for discontinuities in the image.

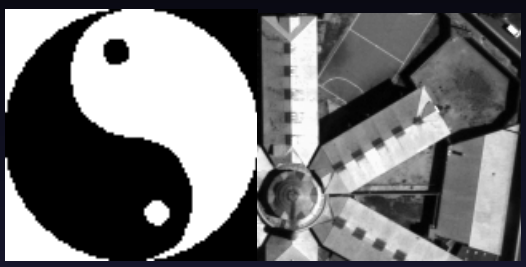

Reference images

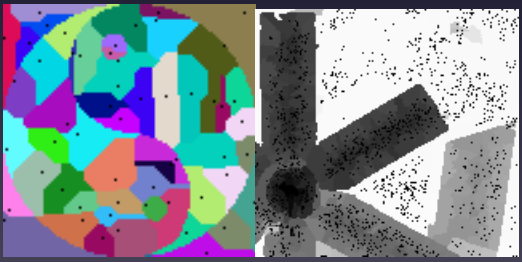

Geodesic Voronoi cells 


\section{Piecewise Affine Interpolation}

- Lambertian hypothesis: a uniform surface with a constant angle has a constant intensity in the image

- Extrapolate information across uniform regions

- Only one sample per cell: Piecewise constant

\section{Geodesic neighborhood}

$\operatorname{GN}_{K}(p)$ of $p$, set of $K$-nearest (geodesic) samples of $\Lambda$ to $p$.

Obs: Samples in $G N_{K}(p)$ likely to have the same model as $p$.
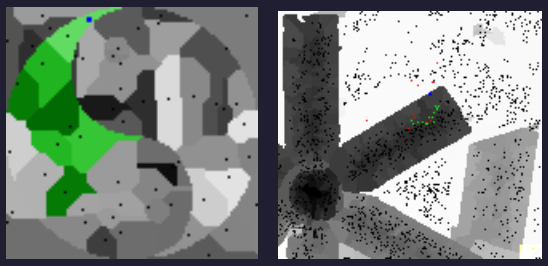

Geodesic neighborhood 


\section{Piecewise Affine Interpolation}

Robust affine plane interpolation using $G N_{K}$

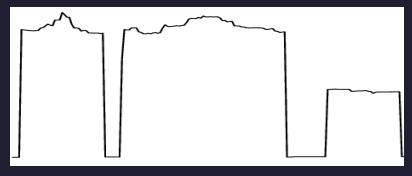

Linear blend with 5 GNN

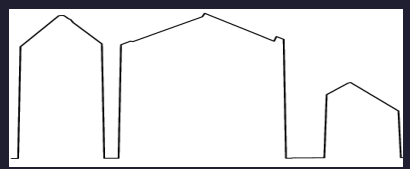

Affine estim. same 5 GNN
- $\forall p \in \wedge$ use RANSAC to fit an affine plane trough $G N_{K}(p)$

- Then extended the model to the entire cell

- We call $H$ the piecewise affine model union of all the cells

- Attention: this is noiseless data 


\section{Constrained Region Merging}

\section{Oversegmentation problems}

- The model of each cell is independently estimated

- Adjacent cells may end up with different models

Merge regions with compatible models using Mumford-Shah:

$$
E(B, f)=\sum_{R \in \mathcal{P}(\Omega, B)} R \operatorname{Err}(R, f)+\lambda \int_{B} g(s) d s, \quad \lambda \geq 0
$$

- Error term: $R E r r(X, f)=\sum_{X \in X \cap \Lambda}\left|f_{X}(x)-H(x)\right|^{2}$ $H$ : initial plane interpolation, $f_{X}$ : affine model for region $X$

- Boundary lenght: $g(s)$ big at poorly contrasted boundaries, small at well contrasted ones

- Constrained with LSD segments [Grompone et al. '10]

- Computed with greedy algorithm [Morel \& Solimini '95] 


\section{Region Merging}

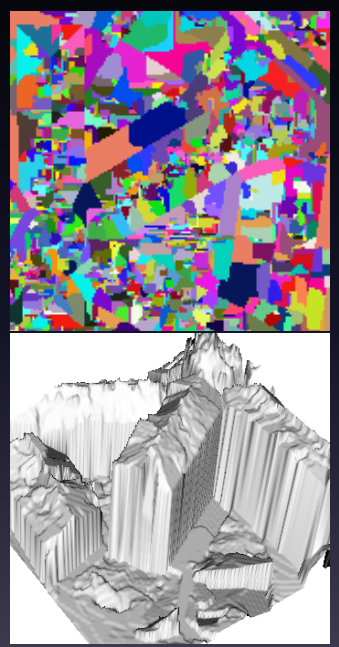

Before merging

V. Caselles and G. Facclolo (UPF) Geodesic Neignoornood interpolation 


\section{Region Merging}

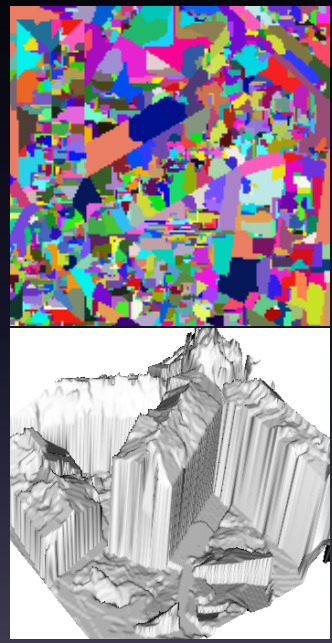

Before merging

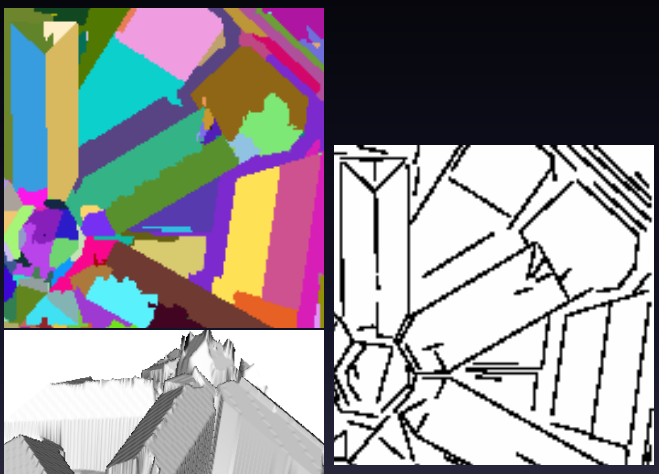

Segment constraints 


\section{Results}

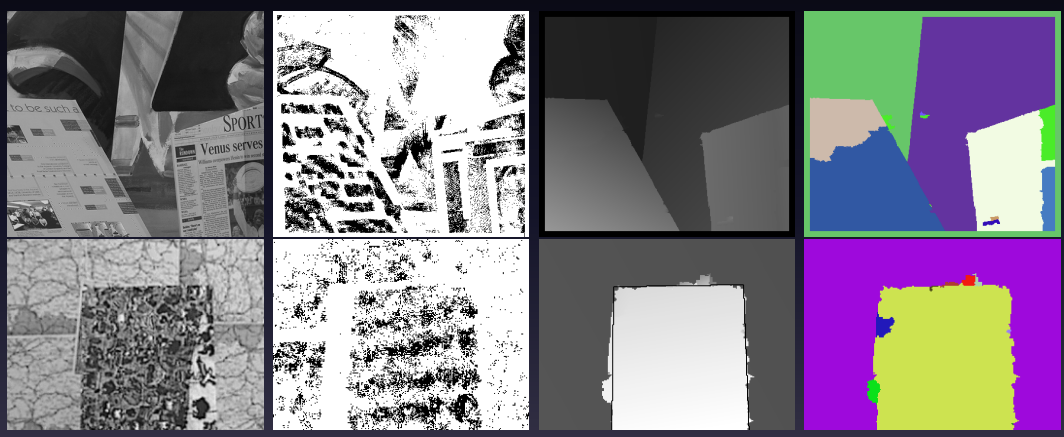




\section{Results}
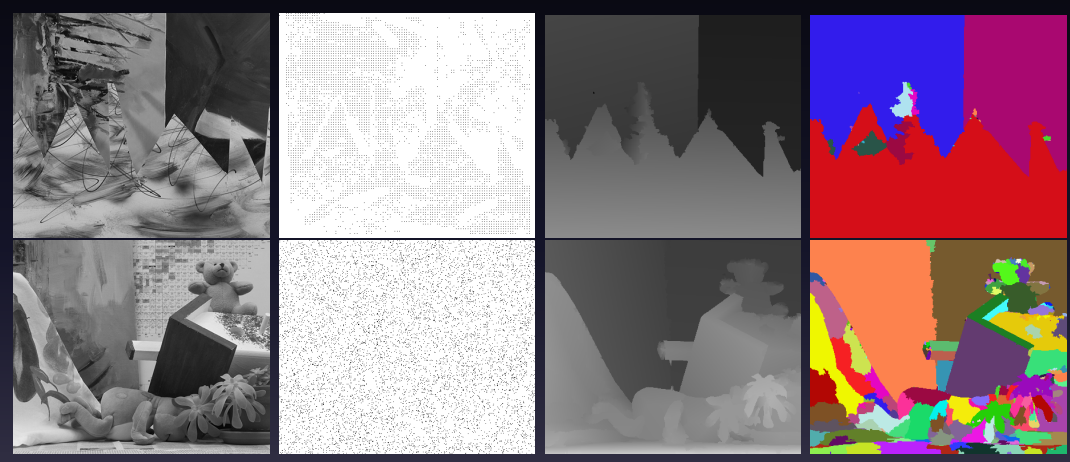

More results at http://gpi.upf.edu/static/geoint 


\section{Conclusions}

- Method for interpolating range data that incorporates geometric information from an image of the scene

- Geodesic neighborhoods: tool for modelling the local information, can be adapted to other (non-affine) models

- Poor results for badly contrasted edges between strongly textured regions 


\section{Conclusions}

- Method for interpolating range data that incorporates geometric information from an image of the scene

- Geodesic neighborhoods: tool for modelling the local information, can be adapted to other (non-affine) models

- Poor results for badly contrasted edges between strongly textured regions

Future work?

- Incorporate texture descriptors to geodesic distance [Bai \& Sapiro '07]

- Improve region merging, tree of mergings [Cardelino '10] 


\section{Conclusions}

- Method for interpolating range data that incorporates geometric information from an image of the scene

- Geodesic neighborhoods: tool for modelling the local information, can be adapted to other (non-affine) models

- Poor results for badly contrasted edges between strongly textured regions

Future work?

- Incorporate texture descriptors to geodesic distance [Bai \& Sapiro '07]

- Improve region merging, tree of mergings [Cardelino '10]

\section{Thank you for your attention http://gpi.upf.edu/static/geoint}

